\title{
Disfunção Temporomandibular segundo o Nível de Ansiedade em Adolescentes
}

\author{
Lara Jansiski Motta ${ }^{1}$ \\ Sandra Kalil Bussadori \\ Camila Leal Haddad de Godoy \\ Daniela Aparecida Biazotto-Gonzalez \\ Universidade Nove de Julho \\ Manoela Domingues Martins \\ Universidade Federal do Rio Grande do Sul \\ Rebeca Souza e Silva \\ Universidade Federal de São Paulo
}

\begin{abstract}
RESUMO - O objetivo do estudo foi determinar a prevalência de sinais e sintomas de disfunção temporomandibular (DTM), segundo o nível de ansiedade de adolescentes da cidade de São Roque-SP. Foi utilizado o Índice de Fonseca para determinar a presença e o grau de severidade da DTM. Para avaliar o nível de ansiedade, foi utilizado o Inventário de Ansiedade TraçoEstado. Os participantes foram 3538 adolescentes entre 10 e 19 anos. Os resultados revelaram que 73,3\% dos adolescentes apresentavam DTM e 72,7\%, apresentavam ansiedade. Foram observadas associações estatisticamente significativas entre a presença de DTM e a presença de ansiedade, mas apenas com o sexo feminino, e correlação positiva, embora baixa, entre o grau de DTM e o nível de ansiedade. Conclui-se que adolescentes do sexo feminino apresentam maior chance de desenvolver DTM que os do sexo masculino, e quanto maior o nível de ansiedade do adolescente, maior a chance de desenvolver DTM.
\end{abstract}

Palavras-chave: adolescentes, ansiedade, disfunção temporomandibular

\section{Temporomandibular Disorder According to the Level of Anxiety in Adolescents}

\begin{abstract}
The aim of this study was to determine the prevalence of signs and symptoms of temporomandibular disorder (TMD), according to the level of anxiety in adolescents in the city of São Roque-SP. The Fonseca Index was used to determine the presence and severity of TMD. To assess the level of anxiety the State-Trait Anxiety Inventory it was used. The participants were 3,538 adolescents with ages varying between 10 and 19 years. The results revealed that $73.3 \%$ of the adolescents had TMD, and that a "slight" degree of TMD was the most prevalent category. Anxiety was present in $72.7 \%$ of the adolescents. The analysis showed the existence of a significant association among female students between the presence of TMD and anxiety. A significant positive correlation was observed between the degree of TMD and the level of anxiety. The results indicate that female adolescents have a higher chance of developing TMD than male adolescents, and that the chance of developing TMD augments with the level of anxiety.
\end{abstract}

Keywords: adolescents, anxiety, temporomandibular disorder

A disfunção temporomandibular (DTM), segundo a Academia Americana de Dor Orofacial (American Academy of Orofacial Pain, 2009), é um termo designado a um subgrupo de dores orofaciais, cujos sinais e sintomas incluem dor ou desconforto na articulação temporomandibular (ATM), nos ouvidos, nos músculos mastigatórios de um ou ambos os lados, nos olhos, na face, nas costas e região cervical. Essa sintomatologia variada está associada a características anatômicas que entram em desequilíbrio (Ferreira, Guimarães, Batista, Ferraz-Júnior, \& Ferreira, 2009). Observa-se também a presença de ruídos articulares, limitação de movimento mandibular e de mastigação (Okeson, 2000).

O estudo da prevalência de sinais e sintomas de DTM tem demonstrado que, na população adulta, essa disfunção pode atingir mais de $75 \%$ dos indivíduos (Egermark, Carlsson, \& Magnusson, 2001; Thilander, Rubio, Pena, \& de Mayorga, 2002). No entanto, há uma carência de estudos na população adolescente. Estudos epidemiológicos como os de Egermark

1 Avenida Getúlio Vargas, 386, Jardim Lourdes, São Roque, SP, Brasil. CEP. 18.130-430.E-mail: larajmotta@terra.com.br et al. (2001) e Thilander et al. (2002) mostraram que sinais e sintomas de DTM podem ser encontrados em todas as idades, com predominância da idade adulta. A maioria dessa população é composta por mulheres, em uma proporção que pode chegar a cinco mulheres para cada homem, e isso tem sido explicado por meio de uma interação de fatores biológicos, hormonais, psicológicos e sociais (Suvinen, Reade, Kemppainen, Könönen, \& Dworkin, 2005), o que reforça a característica multifatorial da DTM.

A etiologia da DTM é complexa e multifatorial (Ferreira et al., 2009; Scarpelli, 2007; Siqueira \& Teixeira, 2001) com relação aos fatores predisponentes, iniciantes e perpetuantes, dentre eles, emoções, traumas, postura e hiperatividade muscular (Okeson, 2000). Tais condições têm curso evolutivo em dias, meses ou anos, ocasionalmente são transitórias e auto-limitantes, agravando-se com hábitos parafuncionais, que são aqueles não relacionados à execução das funções normais do sistema mastigatório (i.e., deglutição, mastigação e fonação), como o bruxismo e o apertamento dental (Ferreira et al., 2009; Scarpelli, 2007; Siqueira \& Teixeira, 2001). A literatura descreve que, dentre os fatores que desencadeiam as DTMs, os de origem psicossomáticas (e.g., depressão 
e ansiedade) devem ser enfatizados, pois condições como a ansiedade podem desencadear hábitos parafuncionais e tensão muscular, levando ao aparecimento dos sinais e sintomas de DTM (Boever, 1981).

A DTM está associada ao conceito do modelo biopsicossocial que considera questões biológicas, psicológicas e sociológicas, não havendo separação entre mente e corpo (Campi, Camparis, Jordani, \& Gonçalves, 2013). Indivíduos com DTM frequentemente apresentam sofrimento psicológico significativo, sendo exemplificado na literatura por distúrbios de humor, elevados índices de ansiedade e de estresse (Burris, Evans, \& Carlson 2010).

A procura de serviços odontológicos por pacientes acometidos pela DTM tem crescido nos últimos anos. Porém, a característica multifatorial dessa disfunção destaca a necessidade de uma tratamento multidisciplinar. Isto é, para a promoção do bem-estar físico, social e emocional do indivíduo, é fundamental que seja feito um trabalho profissional em equipe. Assim, cirurgiões-dentistas, fisioterapeutas, fonoaudiólogos e psicólogos devem conjuntamente avaliar os fatores causais e perpetuantes para intervenção, visando o desenvolvimento de intervenções específicas articuladas e eficazes (Donnarumma, Muzilli, Ferreira, \& Nemr, 2010). A área da psicologia, por exemplo, pode colaborar com o estudo, prevenção e tratamento da DTM direcionando a atenção aos fatores comportamentais e emocionais revelevantes, como aqueles envolvidos no fenômeno da ansiedade.

A ansiedade é percebida como uma emoção caracterizada por um alerta tenso e fisicamente exaustivo, focalizado em um perigo ou emergência iminente e inevitável, embora não objetivamente aparente, com uma incerteza dolorosa sobre a possibilidade de se resolver a situação (Gama, Moura, Araújo, \& Teixeira-Silva, 2008). No estudo da ansiedade, encontramos dois conceitos distintos: a ansiedade-estado, referente a um estado emocional transitório, caracterizado por sentimentos subjetivos de tensão que podem variar em intensidade ao longo do tempo, e a ansiedade-traço referese a uma estrutura relativamente estável e permanente, sendo caracterizada por uma tendência a reagir a situações percebidas como ameaçadoras (Fernandes, Garcia, Zuim, Cunha, \& Marchiori, 2007; Martin, 1998; Spielberger, Gorsuch, \& Lushene, 1970).

Estudos recentes com indivíduos adultos têm mostrado forte relação entre DTM e estados depressivos e ansiosos (e.g., Ferreira et al., 2009; Giannakopoulos, Keller, Rammelsberg, Kronmüller, \& Schmitter., 2010; Monteiro, Zuim, Pesqueira, Ribeiro, \& Garcia, 2011). No entanto, há algumas divergências nos resultados dessas pesquisas. Por exemplo, Ferreira et al. (2009) e Monteiro et al. (2011) indicaram que essa relação é mais provável no sexo feminino, o que não foi observado por Giannakopoulos et al. (2010). Essa inconsistência pode ser explicada, em parte, pelo fato de que, nesse último estudo, o número de homens foi menor que a metade do número de mulheres participantes, o que aponta a necessidade de investigações que tenham representação populacional em número amostral e distribuição. A literatura também tem indicado que a ansiedade pode levar à exacerbação dos sintomas da DTM e modificar a percepção da dor (Ferreira et al., 2009; Giannakopoulos et al., 2010; Monteiro et al., 2011; Okeson, 2000).

A alta prevalência de DTM e suas consequências negativas para a qualidade de vida dos indivíduos (e.g., dor crônica) demanda um diagnóstico precoce dessa disfunção (Okeson, 2000). Investir em estudos com indivíduos mais jovens, como os adolescentes, poderia favorecer a elaboração de protocolos de atendimento e intervenção precoces, contribuindo para o desenvolvimento saudável desses adolescentes. Apesar disso, não são frequentes estudos sobre DTM nessa faixa etária. Um deles foi realizado por Marchiori, Garcia, Zuim e Cunha (2007). Nesse estudo foram avaliados alunos do Ensino Fundamental, entre 9 e 15 anos, sendo observado que o grau de DTM foi mais elevado no sexo feminino. Em outro estudo, Cunha, Fernandes, Marchiori, Garcia e Zuim (2007) compararam a presença de DTM e ansiedade em adolescentes do ensino médio e na fase pré-vestibular e concluíram que tanto a presença de DTM, quanto de ansiedade, foi maior no grupo da fase pré-vestibular, sugerindo forte relação entre DTM e ansiedade. Esses estudos indicam que a DTM é mais provável, em se tratando de adolescentes mais jovens, nas meninas, e que há forte associação entre DTM e ansiedade em adolescentes mais velhos. Uma questão que se segue é se a associação DTM-ansiedade seria observada já no início da adolescência e se seria mais provável entre adolescentes do sexo feminino.

Diante desse quadro, o presente estudo avaliou, em adolescentes, a prevalência de DTM e ansiedade, com ênfase na investigação de possíveis relações entre ambas, utilizando os instrumentos de avaliação IDATE e Índice de Fonseca. Também foi verificado a relevância da variável sexo para a relação DTM-ansiedade. Foram avaliados 3538 adolescentes, estudantes da rede pública de ensino, sem seleção prévia quanto ao estado de saúde, características que conferem originalidade à presente pesquisa, uma vez que os estudos publicados, além de majoritariamente realizados com adultos (e.g., Donnarumma et al., 2010), apresentam um número amostral menor (e.g., Fernandes et al., 2007) e são, na maioria, realizados em centros de tratamento da DTM (e.g., Donnarumma et al., 2010), o que indica que os participantes já estão em tratamento. Por fim, o presente estudo pretende despertar a atenção para a necessidade do envolvimento do psicólogo no tratamento multidisciplinar da DTM.

\section{Método}

O projeto de pesquisa foi aprovado pelo Comitê de Ética em Pesquisa da Universidade Federal de São Paulo UNIFESP (Processo 0886/10).

\section{Participantes}

Participaram do estudo 3538 adolescentes regularmente matriculados, no ano de 2010, no ensino fundamental da rede pública de ensino da cidade de São Roque - SP. Os participantes tinham entre 10 e 19 anos (Média=13,03 anos; $D P=1,57$ ), faixa etária estabelecida pela Organização Mundial de Saúde - OMS (World Health Association - 
WHO, 1986) como definidora da adolescência, sendo 50,9\% $(n=1800)$, do sexo masculino e $49,1 \%(n=1738)$ do sexo feminino.

\section{Instrumentos}

Para avaliação dos sinais e sintomas da DTM e da ansiedade, foram selecionados dois instrumentos: o Índice de Fonseca e o IDATE, respectivamente.

Índice de Fonseca. Esse índice anamnésico, desenvolvido por Fonseca, Bonfante, Valle e Freitas (1994), foi selecionado para este estudo porque é adaptado à população brasileira, é de fácil compreensão e aplicação e pode ser utilizado em várias faixas etárias. $\mathrm{O}$ instrumento inclui informações a respeito de dificuldades em abrir a boca e movimentar a mandíbula; dores na cabeça, nuca, pescoço ou regiões articulares; ruído nas articulações temporomandibulares; hábito de apertar ou ranger os dentes. É composto por 10 perguntas para as quais as possíveis respostas são sim (10 pontos), às vezes ( 5 pontos) e não ( 0 pontos). Para cada pergunta, somente pode ser assinalada uma resposta. A soma de pontos é usada para classificar a severidade da DTM: severa (70 a 100 pontos), moderada (45 a 65), leve (20 a 40) e sem disfunção (0 a 15).

Inventário de Ansiedade Traço-Estado - IDATE (do inglês State-Trait Anxiet Inventory - STAI). Esse instrumento foi traduzido para o português e validado para brasileiros por Biaggio e Natalício (1979). É um dos mais utilizados para avaliar ansiedade por ser de fácil compreensão e aplicação (Andrade, Gorenstein, Vieira Filho, Tung, \& Artes, 2001; Gama et al., 2008; Gorenstein \& Andrade, 1996). Esse inventário é constituído de 40 afirmações a respeito dos sentimentos do sujeito, distribuídas em duas partes. A primeira parte avalia a ansiedade-estado, enquanto a segunda avalia a ansiedade-traço. Cada parte consiste de 20 afirmações descritivas de sentimentos pessoais, as quais os indivíduos pontuam com base na intensidade da ansiedade que está ocorrendo naquele momento (ansiedade-estado) ou com base na frequência com esses sentimentos ocorrem (ansiedade-traço), por meio de uma escala que varia de $1 \mathrm{a}$ 4 pontos.

Os escores obtidos do IDATE são comparados com aqueles pré-determinados pelo questionário, conforme os níveis de ansiedade e suas consequências (Biaggio \& Natalício, 1979; Fernandes et al., 2007):

- Ansiedade baixa ou branda (de 20 a 34 pontos): torna a pessoa alerta, aumentando sua percepção. Este nível de ansiedade pode ser benéfico, pois pode motivar o aprendizado e produzir criatividade.

- Ansiedade moderada (de 35 a 49 pontos): leva a pessoa a focalizar as preocupações imediatas e bloquear as preocupações secundárias, estreita o campo de concentração, gera desatenção seletiva, mas não afeta a capacidade da pessoa focalizar mais áreas, caso se disponha a isso.

- Ansiedade elevada ou grave (de 50 a 64): reduz muito o campo de percepção, de modo que a pessoa focaliza um detalhe específico e não pensa a respeito de outros fatos; induz a pessoa a dirigir seu comportamento para a obtenção de alívio, precisando de muita orientação para focalizar sua atenção.
- Ansiedade muito elevada ou pânico (de 65 a 80): associase ao pavor, terror e medo. Gera desprezo desproporcional pelos detalhes, perda de controle e incapacidade de desenvolver atividades mesmo com alguém orientando verbalmente os passos para o desenvolvimento da atividade. O pânico envolve a desorganização da personalidade, aumenta a atividade motora, diminui a capacidade de comunicação, produz percepções distorcidas e perda do pensamento racional; sendo incompatível com a vida, resulta em exaustão e morte se for mantido por tempo prolongado.

Esse instrumento já foi utilizado com adolescentes por Araújo, Lima-Pereira e Kac (2007), Baptista, Baptista e Torres (2006) e Silva e Koller (2009).

\section{Procedimentos}

Após autorização e assinatura do Termo de Consentimento Livre e Esclarecido, os participantes responderam a dois questionários autoexplicativos para rastreamento de diagnóstico de DTM e ansiedade. Os questionários foram aplicados pela mesma pesquisadora, previamente treinada para auxiliar os alunos apenas em caso de dúvidas em relação ao significado das palavras que compõem os questionários. A aplicação foi realizada em grupo, na sala de aula dos estudantes.

A avaliação de sinais e sintomas da DTM foi obtida por meio do Índice de Fonseca e a avaliação da ansiedade, por meio do IDATE. Inicialmente aplicou-se o IDATE e, logo em seguida, o índice de Fonseca. A aplicação de cada instrumento demorou cerca de 20 minutos. Neste estudo foi utilizado apenas o questionário ansiedade-traço, ou seja, não foi considerado o estado transitório, mas sim, a propensão individual, pois a DTM é considerada uma doença crônica e não uma alteração transitória. Os questionários foram respondidos fora de situações que pudessem provocar ansiedade, como provas ou seminários, para que não houvesse interferência de tais situações sobre os dados coletados, conforme proposto por Fernandes et al. (2007).

\section{Análise de dados}

Os participantes foram avaliados considerando o grau de DTM obtido pelo Índice de Fonseca e o nível de ansiedade encontrado pelo IDATE. Para a análise da associação entre a presença de DTM e a presença de ansiedade, utilizou-se o teste qui-quadrado $\left(\chi^{2}\right)$, e para a análise da correlação entre o grau de DTM e o nível de ansiedade, aplicou-se o teste de correlação de Spearman. Por fim, calculou-se a razão de chance (do inglês odds ratio - $O R$ ) para verificar a possibilidade de homens ou mulheres apresentarem maior ou menor chance de desenvolver DTM. Em todas as análises foi considerado um nível de significância de $5 \%$.

\section{Resultados}

A Tabela 1 mostra a incidência de DTM entre os adolescentes segundo o sexo. Dos 3538 participantes 
avaliados, 73,3\% relataram algum grau de DTM. Destes, $80,7 \%$ eram do sexo feminino e $66,0 \%$, do sexo masculino, sendo essa diferença entre sexos estatisticamente significativa $\left(\chi^{2}=98,98 ; \mathrm{p}<0,001\right)$.

Quando avaliados os diferentes graus de DTM de acordo com o sexo (ver Tabela 2), observa-se que 58\%

Tabela 1. Frequência absoluta e relativa de adolescentes com disfunção temporomandibular (DTM) segundo o sexo

\begin{tabular}{cccc}
\hline \multirow{2}{*}{ Sexo } & \multicolumn{2}{c}{ DTM } & \multirow{2}{*}{ Total geral } \\
\cline { 2 - 3 } & Sem DTM & Com DTM & \\
\hline Masculino & $612(34,0 \%)$ & $1188(66,0 \%)$ & 1800 \\
Feminino & $336(19,3 \%)$ & $1402(80,7 \%)$ & 1738 \\
Total & $948(26,8 \%)$ & $2590(73,2 \%)$ & 3538 \\
\hline
\end{tabular}

dos participantes apresentaram disfunção "leve", 14,1\%, disfunção "moderada" e apenas 1,2\%, disfunção "severa" (total de 73,2\% de adolescentes com DTM). Além disso, os graus moderado e severo foram mais frequentes no sexo feminino $(63,3 \%$ e $63,4 \%$, respectivamente) do que no sexo masculino $(36,7 \% 336,6 \%$, respectivamente). Essas diferenças entre sexos foram estatisticamente significativas e sugerem que a DTM apresenta maior severidade entre as meninas $\left(\chi^{2}=119,702, \mathrm{p}<0,001\right)$. A Tabela 3 indica a ou muito elevada); dentre aqueles sem DTM (948), não mais do que 50,8\% apresentaram ansiedade. Essa diferença entre participantes com e sem DTM foi estatisticamente significativa $\left(\chi^{2}=314,59, \mathrm{p}<0,001\right)$.

A Tabela 5 mostra a frequência de adolescentes com diferentes graus de DTM em função dos níveis de ansiedade. Houve uma correlação positiva entre graus de DTM e níveis de ansiedade. Por exemplo, 55,8\% dos participantes com

Tabela 4. Frequência absoluta e relativa de adolescentes com disfunção temporomandibular (DTM) segundo o nível de ansiedade

\begin{tabular}{cccc}
\hline \multirow{2}{*}{ DTM } & \multicolumn{2}{c}{ Ansiedade } & Total geral \\
\cline { 2 - 3 } & Normal & $\begin{array}{c}\text { Elevados } \\
\text { Níveis }\end{array}$ & \\
\hline Sem DTM & $466(49,2 \%)$ & $482(50,8 \%)$ & 948 \\
Com DTM & $497(19,2 \%)$ & $2093(80,8 \%)$ & 2590 \\
Total & $963(27,2 \%)$ & $2575(72,7 \%)$ & 3538 \\
\hline
\end{tabular}

DTM "leve" apresentaram ansiedade "moderada" e 53,7\% $(n=22)$ daqueles com DTM "severa" apresentaram ansiedade "elevada". A correlação obtida foi de baixa magnitude $(r=0,401)$, mas estatisticamente significativas $(p<0,0001)$.

Considerando a maior prevalência de DTM e ansiedade no sexo feminino, analisou-se a razão de chance (OR) que

Tabela 2. Frequência absoluta e relativa de adolescentes com diferentes graus de disfunção temporomandibular (DTM) segundo o sexo

\begin{tabular}{|c|c|c|c|c|c|}
\hline \multirow{2}{*}{ Sexo } & \multicolumn{4}{|c|}{ DTM } & \multirow{2}{*}{ Total Geral } \\
\hline & Sem DTM & Leve & Moderada & Severa & \\
\hline Masculino & $612(64,6 \%)$ & $990(48,3 \%)$ & $183(36,7 \%)$ & $15(36,6 \%)$ & \\
\hline Feminino & $336(35,4 \%)$ & $1061(51,7 \%)$ & $315(63,3 \%)$ & $26(63,4 \%)$ & \\
\hline Total & $948(26,7 \%)$ & $2051(58 \%)$ & $498(14,1 \%)$ & $41(1,2 \%)$ & 3538 \\
\hline
\end{tabular}

frequência de adolescentes com diferentes níveis de ansiedade conforme o sexo. Observa-se que 51,4\% dos participantes relataram ansiedade moderada, 19,5\%, ansiedade elevada ou grave, e 1,8\%, ansiedade muito elevada (total de $72,7 \%$ de adolescentes ansiosos). Níveis elevados e muito elevados de ansiedade foram mais comuns entre adolescentes do sexo feminino (82,9\% e 69,3, respectivamente) do que do sexo masculino (17,1\% e 30,7\%, respectivamente), uma diferença apontada como estatisticamente significativa $\left(\chi^{2}=267,783\right.$, $\mathrm{p}<0,001)$. A relação entre a presença de DTM e os níveis de ansiedade é mostrada na Tabela 4. Independentemente do sexo, dos 2590 participantes que apresentaram DTM, 80,8\% apresentaram algum nível de ansiedade (moderada, elevada participantes do sexo feminino têm, em relação aos do sexo masculino, de apresentarem DTM, assim como de que participantes mais ansiosos também apresentem essa disfunção. Essa análise é mostrada na Tabela 6 . O valor da OR igual a 1,508 indica que adolescentes do sexo feminino apresentaram uma chance 1,5 maior que adolescentes do sexo masculino de ter DTM, um resultado com significância estatística $(I C 95 \%=1,278-1,777)$. Adicionalmente, o valor da $O R$ igual a 3,103 sugere que adolescentes com níveis mais elevados de ansiedade têm três vezes mais chance de ter DTM do que adolescentes com nível de ansiedade branda ou normal. Esse resultado foi estatisticamente significativo $($ IC $95 \%=2,728-3,528)$.

Tabela 3. Frequência absoluta e relativa de adolescentes com diferentes níveis de ansiedade segundo o sexo

\begin{tabular}{cccccc}
\hline Sexo & Normal & Moderada & Elevada & $\begin{array}{c}\text { Muito } \\
\text { Elevada }\end{array}$ & Total \\
\hline Masculino & $664(68,9 \%)$ & $911(50,1 \%)$ & $11(17,1 \%)$ & $214(30,7 \%)$ & \\
Feminino & $299(31,1 \%)$ & $907(49,9 \%)$ & $53(82,9 \%)$ & $479(69,3 \%)$ & \\
Total & $963(27,3 \%)$ & $1818(51,4 \%)$ & $64(19,5 \%)$ & $693(1,8 \%)$ & 3538 \\
\hline
\end{tabular}


Tabela 5. Frequência absoluta e relativa de adolescentes com diferentes graus de disfunção temporomandibular (DTM) em relação aos niveis de ansiedade

\begin{tabular}{|c|c|c|c|c|c|}
\hline \multirow[b]{2}{*}{ DTM } & \multicolumn{4}{|c|}{ Ansiedade } & \multirow[b]{2}{*}{ Total } \\
\hline & Normal & Moderada & Elevada & $\begin{array}{c}\text { Muito } \\
\text { Elevada }\end{array}$ & \\
\hline Sem DTM & $469(49,5 \%)$ & $430(45,4 \%)$ & $47(5,0 \%)$ & $2(0,21 \%)$ & 948 \\
\hline Leve & $464(22,6 \%)$ & $1145(55,8 \%)$ & $412(20,1 \%)$ & $30(1,5 \%)$ & 2051 \\
\hline Moderada & $33(6,6 \%)$ & $231(46,4 \%)$ & $209(42,0 \%)$ & $25(5,0 \%)$ & 498 \\
\hline Severa & $0(0 \%)$ & $12(29,3 \%)$ & $22(53,7 \%)$ & $7(17,1 \%)$ & 41 \\
\hline Total & $963(27,3 \%)$ & $1818(51,4 \%)$ & $690(19,5 \%)$ & $64(1,8 \%)$ & 3538 \\
\hline
\end{tabular}

Tabela 6. Frequência absoluta e relativa de adolescentes com diferentes graus de disfunção temporomandibular (DTM) em relação aos níveis de ansiedade

\begin{tabular}{ccccc}
\hline Variáveis & $\boldsymbol{p}$ & OR & \multicolumn{2}{c}{ IC (95\%) } \\
\hline Sexo feminino x DTM & $<0,001$ & 1,508 & 1,278 & 1,777 \\
Grau de ansiedade x DTM & $<0,001$ & 3,103 & 2,728 & 3,528 \\
\hline
\end{tabular}

\section{Discussão}

O objetivo desta pesquisa foi analisar a prevalência de DTM e ansiedade, e suas possíveis relações. Os resultados mostraram que a prevalência de DTM e ansiedade é elevada na adolescência e que existe uma correlação positiva entre o grau de DTM e o nível de ansiedade. Foi também observado que adolescentes do sexo feminino apresentam maior chance de desenvolver DTM que os do sexo masculino, e que níveis altos de ansiedade do adolescente aumentam a chance de desenvolver DTM em três vezes.

Quanto à prevalência, verificou-se que, de acordo com o Índice de Fonseca, dos 3538 adolescentes avaliados, $73,3 \%$ apresentaram algum grau de DTM. Essa prevalência apresentou-se um pouco mais elevada do que na maioria dos estudos de DTM com adultos (e.g., Ferreira et al., 2009; Monteiro et al., 2011), os quais descreveram uma prevalência de $30 \%$ a $60 \%$, mas concordam com estudos epidemiológicos, como o de Egermark et al. (2001) e Thilander et al. (2002), que demonstraram que a prevalência pode chegar a $75 \%$ na mesma população. A alta prevalência de DTM na adolescência, aqui verificada, chama a atenção para a necessidade do diagnóstico precoce, de modo a minimizar problemas futuros relacionados à dor crônica e suas consequências na qualidade de vida do indivíduo (Ferreira et al., 2009).

No grupo de adolescentes estudados neste trabalho, $80,7 \%$ das meninas apresentaram DTM, enquanto entre os meninos, apenas $66 \%$ mostraram DTM. Esse resultado é consistente com aqueles relatados em investigações com adultos, os quais apontam a DTM como uma doença que atinge mais as mulheres (Dworkin \& LeResche, 1992; Egermark et al., 2001; Monteiro et al., 2011; Oliveira, Dias, Contato, \& Berzin, 2006; Suvinen et al., 2005). Essa diferença entre os sexos pode ser devida a fatores fisiológicos, tais como variações hormonais e estrutura muscular, mas essa possibilidade ainda não foi explicitamente investigada. Apesar da metodologia aplicada nesta pesquisa não permitir a identificação do motivo da diferença entre sexos, os resultados servem de subisídio para a justificativa de estudos clínicos que comparem, entre meninos e meninas, níveis hormonais, estrutura física muscular e diferenças anatômicas relacionadas à DTM. Pesquisas clínicas poderão contribuir para o desenvolvimento de protocolos de tratamento e programas preventivos de DTM específicos para adolescentes.

A presente pesquisa também fornece evidências de que a ansiedade deve ser considerada tanto no diagnóstico, quanto no tratamento e acompanhamento da DTM. Dentre os adolescentes com DTM, 80,8\% mostraram-se ansiosos. Essa alta prevalência de ansiedade também foi encontrada em estudos de ansiedade-traço com jovens universitários com idade superior a 18 anos (Dworkin \& LeResche, 1992; Egermark et al., 2001; Monteiro et al., 2011; Oliveira et al., 2006). Em conjunto, esses resultados sugerem que a relação DTM-ansiedade pode estar presente desde o início da adolescência e se estender para a vida adulta, o que reforça a necessidade de realizar um diagnóstico precoce e multidisciplinar.

Possivelmente, essa relação entre DTM e ansiedade ocorre porque a dor desencadeada pela DTM pode gerar reações ansiosas que, por sua vez, podem diminuir o limiar à dor (Vedolin, 2007). Borini, Duarte, Amorim e Bérzin (2010) e Hoehn-Saric e McLeod (2000) realizaram estudos eletromiográficos da atividade muscular e concluíram que indivíduos ansiosos apresentaram maior tensão muscular, o que pode causar dor. Uma vez que dentre os sinais e sintomas da DTM, a dor muscular é muito frequente, principalmente nos músculos mastigatórios (Casanova-Rosado et al., 2006; Ferreira et al., 2009; Okeson, 2000; Scarpelli, 2007), e já que a ansiedade pode promover dor muscular, tratamentos de DTM devem incluir estratégias para lidar com ansiedade.

A verificação de uma correlação positiva, embora baixa, entre o grau de DTM e o nível de ansiedade (com o aumento do nível de ansiedade, a DTM apresentou-se mais severa) reforça a proposta de que aspectos psicológicos devem ser enfatizados no tratamento da DTM. Segundo Rezende, Silva, Camargos e Barbosa (2010), que relataram a experiência de um atendimento conjunto entre odontologia e psicologia em pacientes com DTM, a associação entre terapêuticas contribui para a redução da severidade da DTM e redução dos níveis de ansiedade. Tratamentos similares poderiam ser aplicados em adolescentes.

Em suma, os resultados da presente pesquisa, em conjunto com aqueles presentes na literatura, sugerem que profissionais da área da odontologia, fonoaudiologia e fisioterapia não podem proporcionar um tratamento integral e resolutivo da DTM, sendo necessária a participação do 
psicólogo no diagnóstico, tratamento e desenvolvimento de pesquisas sobre o tema.

\section{Referências}

American Academy of Orofacial Pain (2009). American Academy of Orofacial Pain Guidelines. Retrieved from http://www. aaop.org

Andrade, L., Gorenstein, C., Vieira Filho, A. H., Tung, T. C., \& Artes, R. (2001). Psychometric properties of the Portuguese version of the State-Trait Anxiety Inventory applied to college students: Factor analysis and relation to the Beck Depression Inventory. Brazilian Journal of Medical and Biological Research, 34(3), 367-374.

Araújo, D. M. R., Lima-Pereira, N., \& Kac, G. (2007). Ansiedade na gestação, prematuridade e baixo peso ao nascer: uma revisão sistemática da literatura. Cadernos de Saúde Pública, 23(4), 747-756.

Baptista, M. N., Baptista, A. S. D., \& Torres, E. C. R. (2006). Associação entre suporte social, depressão e ansiedade em grávidas. Psic: Revista de Psicologia da Vetor Editora, 7(1), $39-48$.

Biaggio, A., \& Natalício, L. F. (1979). Manual do IDATE. Rio de Janeiro: CEPA.

Boever, J. A. (1981). Functional disturbances of the temporomandibular joint. In G. A. Zarb \& G. E. Carlsson (Eds.), The temporomandibular joint. Function and dysfunction (pp. 193-214). Copenhagen: Munksgaard.

Borini, C. B., Duarte, C. L., Amorim, M. M., \& Bérzin, F. (2010). Análise da influência da ansiedade sobre o sinal eletromiográfico. Revista Gaúcha de Odontologia (Online), 58(2), 225-230.

Burris, J. L., Evans, D. R., \& Carlson, C. R. (2010). Psychological correlates of medical comorbidities in patients with temporomandibular disorders. Journal of the American Dental Association (1939), 141(1), 22-31.

Campi, L. B., Camparis, C. M., Jordani, P. C., \& Gonçalves, D. A. D. G. (2013). Influência de abordagens biopsicossociais e autocuidados no controle das disfunções temporomandibulares crônicas. Revista Dor, 14(3), 219-222.

Casanova-Rosado, J. F., Medina-Solís, C. E., Vallejos-Sánchez, A. A., Casanova-Rosado, A. J., Hernández-Prado, B., \& Ávila-Burgos, L. (2006). Prevalence and associated factors for temporomandibular disorders in a group of Mexican adolescents and youth adults. Clinical Oral Investigations, 10(1), 42-49.

Cunha, L. D. A. P., Fernandes, A. Ú. R., Marchiori, A. V., Garcia, A. R., \& Zuim, P. R. J. (2007). Níveis de ansiedade e desordem têmporo-mandibular em estudantes do ensino médio e curso pré-vestibular. Revista Brasileira de Odontologia, 64(3), 233-238.

Donnarumma, M. D. C., Muzilli, C. A., Ferreira, C., \& Nemr, K. (2010). Disfunções temporomandibulares: sinais, sintomas e abordagem multidisciplinar. Revista CEFAC, 12(5), 788-794.

Dworkin, S. F., \& LeResche, L. (1992). Research diagnostic criteria for temporomandibular disorders: Review, criteria, examinations and specifications, critique. Journal of Craniomandibular Disorders: Facial \& Oral Pain, 6(4), 301-355.
Egermark, I., Carlsson, G. E., \& Magnusson, T. (2001). A 20-year longitudinal study of subjective symptoms of temporomandibular disorders from childhood to adulthood. Acta Odontologica, 59(1), 40-48.

Fernandes, A. U. R., Garcia, A. R., Zuim, P. R. J., Cunha, L. D. A. P., \& Marchiori, A. V. (2007). Desordem temporomandibular e ansiedade em graduandos de odontologia. Brazilian Dental Science, 10(1), 70-77.

Ferreira, K. D. M., Guimarães, J. P., Batista, C. H. T., Ferraz Júnior, A. M. L., \& Ferreira, L. A. (2009). Fatores psicológicos relacionados à sintomatologia crônica das desordens temporomandibulares - revisão de literatura. Revista Faculdade de Odontologia, 14(3), 262-267.

Fonseca, D. M. D., Bonfante, G., Valle, A. L. D., \& Freitas, S. F. T. D. (1994). Diagnóstico pela anamnese da disfunção craniomandibular. Revista Gaúcha de Odontologia, 42(1), 23-24.

Gama, M. M. A., Moura, G. S., Araújo, R. F., \& Teixeira-Silva, F. (2008). Ansiedade-traço em estudantes universitários de Aracaju. Revista de Psiquiatria, 30(1), 19-24.

Giannakopoulos, N. N., Keller, L., Rammelsberg, P., Kronmüller, K.-T., \& Schmitter, M. (2010). Anxiety and depression in patients with chronic temporomandibular pain and in controls. Journal of Dentistry, 38(5), 369-376.

Gorenstein, C., \& Andrade, L. H. S. G. (1996). Validation of a Portuguese version of the Beck Depression Inventory and the State-Trait Anxiety Inventory in Brazilian subjects. Brazilian Journal of Medical and Biological Research, 29(4), 453-457.

Hoehn-Saric, R., \& McLeod, D. R. (2000). Anxiety and arousal: Physiological changes and their perception. Journal of Affective Disorders, 61(3), 217-224.

Marchiori, A. V., Garcia, A., Zuim, P. R. J., \& Cunha, L. (2007). Relação entre a disfunção temporomandibular e ansiedade em estudantes do ensino fundamental. Pesquisa Brasileira de Odontopediatria e Clínica Integrada, 7(1), 37-42

Martin, P. (1998). Animal models sensitive to anti-anxiety agents. Acta Psychiatrica Scandinavica, 393, 74-80.

Monteiro, D. R., Zuim, P. R. J., Pesqueira, A. A., Ribeiro, P. do P., \& Garcia, A. R. (2011). Relationship between anxiety and chronic orofacial pain of temporomandibular disorder in a group of university students. Journal of Prosthodontic Research, 55(3), 154-158.

Okeson, J. (2000). Tratamento das desordens temporomandibulares e oclusão. Porto Alegre: Artes Médicas.

Oliveira, A. S., Dias, E. M., Contato, R. G., \& Berzin, F. (2006). Prevalence study of signs and symptoms of temporomandibular disorder in Brazilian college students. Brazilian Oral Research, 20(1), 3-7.

Rezende, K. D. P., da Silva, M. R., Camargos, G. D. V., \& Barbosa, A. V. (2010). Diálogos entre a psicologia e a odontologia: atendimento a pacientes com disfunção temporomandibular. Horizonte Cientifico, 4(2), 1-22.

Scarpelli, P. B. (2007). Análise do comportamento de dor em disfunção temporomandibular (Unpublished master's thesis). Pontifícia Universidade Católica de Campinas, Campinas.

Silva, M., \& Koller, S. H. (2009). Grupoterapia cognitivocomportamental para crianças e adolescentes vítimas de abuso sexual. Revista de Saúde Pública, 43(Supl 1), 70-8.

Siqueira, J., \& Teixeira, M. (2001). Dor orofacial: diagnóstico, terapêutica e qualidade de vida. Curitiba: Maio. 
Spielberger, C. D., Gorsuch, R. L., \& Lushene, R. E. (1970). Manual for the State-Trait Anxiety Inventory. Retrieved from http:// ubir.buffalo.edu/xmlui/handle/10477/2895

Suvinen, T. I., Reade, P. C., Kemppainen, P., Könönen, M., \& Dworkin, S. F. (2005). Review of aetiological concepts of temporomandibular pain disorders: Towards a biopsychosocial model for integration of physical disorder factors with psychological and psychosocial illness impact factors. European Journal of Pain, 9(6), 613-633.

Thilander, B., Rubio, G., Pena, L., \& de Mayorga, C. (2002). Prevalence of temporomandibular dysfunction and its association with malocclusion in children and adolescents: An epidemiologic study related to specified stages of dental development. The Angle Orthodontist, 72(2), 146-154.
Vedolin, G. M. (2007). Participação do estresse e ansiedade na alteração do limiar de dor à pressão em pacientes com DTM miogênica: um estudo comparativo (Unpublished doctoral dissertation). Universidade de São Paulo, São Paulo.

World Health Organization [WHO] (1986). Young people's health - a challenge for society: Report of a WHO study group on young people and health for all by the year 2000. Retrieved from http://apps.who.int/iris/handle/10665/41720

Recebido em 15.05.2013

Primeira decisão editorial em 18.06.2014

Versão final em 24.07.2014

Aceito em 30.07.2014 